\title{
The Structure and Hierarchy of Sentence-endings in Tibetan Language *
}

\author{
Li Huaping ** \\ (Yangtze University, China)
}

\begin{abstract}
This paper discusses the structure and hierarchy of sentence-endings in Tibetan language. Tibetan sentence-endings are hierarchical. They can be divided into two levels from the perspectives of structure, distribution and expressive function. The first level comes after the predicate or verb phrase, indicating the category of tense/aspect $/ \mathrm{mood}$ (TAM). The second level, which follows a self-sufficient sentence, mainly expresses the meaning of the speaker's hint, inference, evaluation and attitude to the information. Each level includes several different types of endings, which act on different syntactic categories or manifest different degrees of subjectivity. The lower the degree of correlation between the endings and the information of the self-sufficient sentence is, the higher the corresponding semantic category and the speaker's subjective participation are. Some lower-level endings can also express the grammatical meaning of the higher level in certain context with increasing subjectivity.
\end{abstract}

Keywords: Tibetan sentence-endings, syntactic hierarchy, sentence-completion category, pragmatic category, subjectivity, syntactic sentence, pragmatic sentence

\section{Introduction}

Tibetan sentence-endings are function words or combination of function words located at the end of a sentence to express meaning of grammatical category, also called verb endings, verb suffix, tense/aspect/mood (TAM) suffixes, TAM auxiliaries, final auxiliaries, etc. As early as the last century, a host of domestic scholars (Jin Peng, 1979; Hu Tan, 1999, 2002; Skalbzang Vgyurmed, 2004; Zhou Jiwen, 2003; etc.) have made full research on these

\footnotetext{
* This study is sponsored by the Project of Research Planning Foundation on Humanities and Social Sciences of the Ministry of Education of China (Number: 15YJA740018) and China Scholarship Council (CSC). All the examples in this article are transliterated according to Wylie's "A Standard System of Tibetan Transcription" with the exception that the letter " $\mathrm{R}$ " is transliterated as " $\mathrm{v}$ " instead of an apostrophe, and the example sentences without indicating sources are collected from Hu Tan (1999).

** Dr. Li Huaping: Associate Professor, School of Humanities, Yangtze University, China. His research interests are Sino-Tibetan and ancient Chinese. Email: lihuaping2000@gmail.com, lihuaping2000@ sina.com.
} 


\section{The Structure and Hierarchy of Sentence-endings in Tibetan Language}

endings and reaped huge fruits on the form and function of sentence-endings. Although foreign scholars have different approaches of analysis and expression, their conclusions are basically the same. The fact that there are different distributions, forms and functions in all these varieties of endings which constitute a set of hierarchical ending system has attracted the attention of many scholars (Hu Tan, 1999; Denwood, 1999; Tournadre, 2003; Delancey, 2011; Lim, 2012; Oisel, 2017). The purpose of this paper is to investigate the structure and the hierarchical relationship among the endings from their linear distribution and function. Considering that Tibetan dialects are complex and diverse, here we will take the Lhasa dialect as an example. The sentence-endings are mainly after a predicate verb (and sometimes further expanded after the verb ending), but some of them are also after the adjective predicate. Due to the limitation of space, this paper only discusses the endings after a predicate verb.

\section{The structure and distribution of Tibetan sentence-endings}

Much of the research on Tibetan language in the last century has analyzed the distribution and structure of endings, and come up with basically consistent conclusions. Jin Peng, the first man to analyze the structure of endings in modern terms in China, pointed out that "the way a verb expresses the mood, tense and aspect is to either add an additional component to the verb then plus an auxiliary verb, or to add an auxiliary verb directly after the verb" (1979:148). This overview summarizes the two major types of ending structures. Coincidentally, Delancey $(2011,2018)$ had the same idea, which focuses more on the diachronic source of the structure of endings. He (2018:583) held the view that:

Some Tibetic languages have finite forms which are simple nominalizations, but in all the largest part of the verb paradigm consists of endings of two kinds: erstwhile serial verbs and nominalized sentence + copula constructions. Lhasa verb inflections are of these two kinds. The core set of endings, which encode the Egophoric and Factual categories, originated and are written as a nominalizing suffix -pa/-ba or -gi/-gyi/-kyi, followed by an equational (yin, red) or existential (yod, vdug) copula. The evidential forms were originally serialized verbs, song 'went' and bzhag 'put [someplace]'.

Here, the "nominalized sentence + copula constructions" evolved into the construction of "nominalized suffix followed by an equational or existential copula" after re-analysis, which became the two major types of ending structure together with the auxiliary verbs that originally derived from serialized verbs. Later, $\mathrm{Hu}$ Tan generalized that these structures express various grammatical meanings after a predicate verb as "sentence-endings", and emphasized that "the tense, person and other categories of verb are mainly indicated by them" (1999:82). The research results of these linguists endow us a lot of enlightenment. These endings, according to their distribution after a predicate verb and the way they are 


\section{Li Huaping}

connected, can be ulteriorly divided into the following types which we are going to talk about one by one.

\subsection{Unitary ending}

The first type (marked as END 1) is the single auxiliary verb that comes directly after the past tense of the predicate verb without the aid of additional components. We call this type unitary ending. It has been confirmed by some previous studies that this kind of ending derives from the grammaticalization of the second verb (or serial verb, such as the verb of motion, position, state, process, etc.) in some serialized verb constructions (Delancy, 1991, 2018; Denwood, 1999; Tournadre, 2003). ${ }^{(1)}$ They are used as final verb suffixes much more frequently than as independent verbs in modern Lhasa dialect, and all correlate to tense-aspects. The sentence structure pattern of this type of ending is:

\section{lexical verb stem (past) + auxiliary}

Typical endings include song, byung, shag, tshar: ${ }^{2}$

(1) sgrol dkar dpe mdzod khang-la phyin-song

sgrol dkar library-LOC went-AUX

'Sgrol dkar went to the library.'

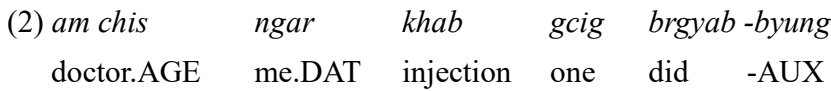

'The doctor gave me an injection.'

\footnotetext{
(1) Serialized verb structure refers to the "VV+ TAM component" structure produced by the shedding of the subordinate marker after the first sentence verb in the double-sentence chain structure. For example, in the double clause (a) kho bros-byas yong-pa red. (he flee-SM came-SPFV, 'He fled, and came hither.'), the subordinate marker byas of the first sentence fell off to form sentence (b) kho bros yong-pa red. (he flee came-SPFV, 'He fled hither.'), whose predicate is a sequence verb structure. According to the degree of grammaticalization, the second verb of this structure can be divided into four categories: (1) Optional serialization such as vgro, yong and sdad, etc. When they are used as the second verb, the subordinate marker after the preceding verb can be omitted, but this case only happens when the first verb is a motion verb of some kind (e.g. 'fly', 'arrive', 'escape', etc.). Otherwise the subordinate marker cannot be omitted; (2) Obligatory serialization. This kind of verbs, such as bzhag, tshar and myong, are used only as the second verb without the subordinate marker on the preceding verb, whose meaning is clearly distinct from the meaning as lexical verbs. However, they also take the same range of TAM inflections as any other verbs; (3) Mixed paradigms. The verb tshar and myong manifest an odd pattern in which they behave as versatile verb in disjunct construction, but in conjunct construction they are more like verb suffixes; (4) Morphologization. Four of these verbs, song, bzhag, byung, and yong, occur unstressed as final verb suffixes, followed by no other TAM components, indicating that they are completely grammaticalized. See Delancey, 1991. Later, Denwood (1999:171-176) refined and modified the classification of Delancey to distinguish five groups representing five increasing degrees of grammaticalization.

(2) As shown in the comment (1), the levels of grammaticalization of the second verb in different contexts are different, so some of them are followed by TAM components in some sentences. For example: (a) kho phyin tshar-pa red. (he went finish-SPFV, 'He has gone.'); (b) kho phyin bzhag-pa red. (he went put-SPFV, 'He went.' [with some negative consequence of his having gone]); (c) kho bod-la vgro myong-pa red. (he Tibet-LOC go experience-SPFV, 'He has been to Tibet.'). In this case they are second verbs rather than endings.
} 


\section{The Structure and Hierarchy of Sentence-endings in Tibetan Language}

$$
\begin{array}{rll}
\text { (3) (ngas) } & \text { yige } & \text { bris -tshar } \\
\text { I.AGE } & \text { letter } & \text { wrote-AUX }
\end{array}
$$

'I have finished writing the letter.'

\subsection{Joint ending}

The second type always appears with additional components, that is, this type is composed of additional components and auxiliary verbs. The additional components are often referred to as nominal suffixes, and the auxiliary verbs are the grammaticalization of copulas. Therefore, this type is also called "nominal suffix followed by copula" by Delancey (2018:583). Here, we call it the joint ending. The sentence structure pattern of this type of ending is:

\section{lexical verb stem + additional component + auxiliary}

For the sake of exposition, we classify this type into two subclasses according to the type of the additional component and semantic function.

\subsubsection{TAM ending, additional components are gi, pa, grabs}

The first one (marked as END 2) consists of an additional component gi/pa/grabs and an auxiliary verb yin/red or yod/vdug/yovo red, such as gi yin, gi yod, gi vdug, pa yin, pa red, grabs yin and so on. These endings are primarily used to express various categories of tenses and evidentials. Of these endings, pa yod and pa vdug are exceptions. Although they also belong to this type of structure and relate to a future event, their primary function is to make an inference from available evidence to express a high degree of certainty. Jiang Di (2005) referred to these two endings as the "contextual aspect". This kind of endings is the most common form in Tibetan. Unlike many other languages, there are two forms of narratives including the special statement and the general statement in Tibetan declarative sentences. ${ }^{(1)}$ Among them, the auxiliary verbs of special statement sentences have the distinction between the self-referential and the other-referential, which are represented by different auxiliary verbs. For instance:

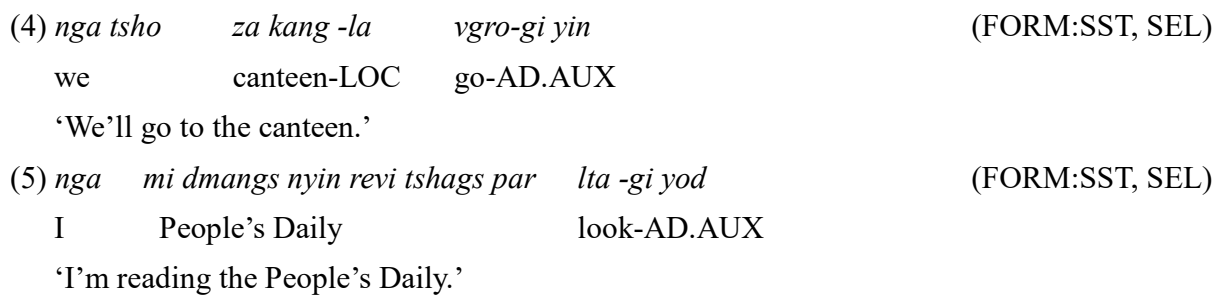

\footnotetext{
(1) Jin Peng (2001:256) has talked about the expression forms of tone (or mood) in modern spoken Tibetan. He pointed out that there are six forms of tones in Tibetan verbs: special statement, general statement, judgment, inference, conjecture and imperative. Among them, the special statement is related to whether the action expressed is known and affirmed by the narrator, or whether it can be the tone of the narrator's subjective decision, whereas the general statement just speaks generally.
} 


\section{Li Huaping}

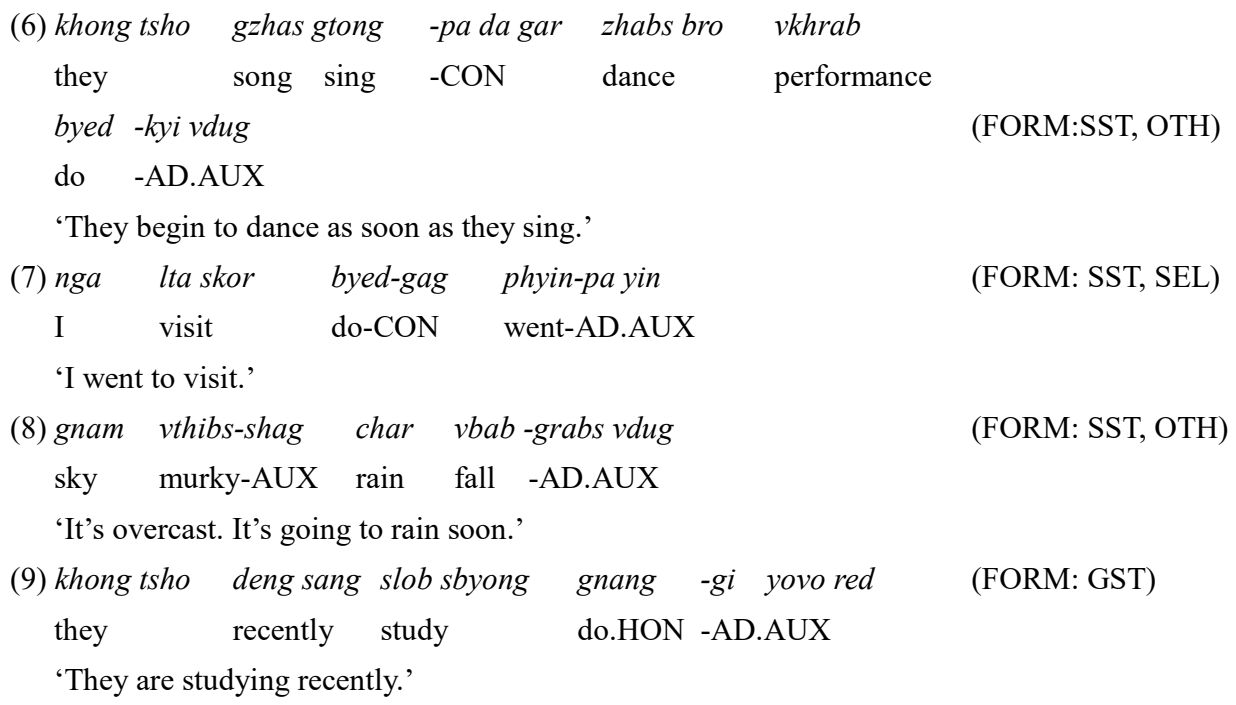

The examples (4)-(8) are special statement sentences, which manifest person difference of the subject by the difference in the auxiliary verb (but in some sentences the auxiliary verb does not completely agree with the subject person). Generally speaking, yin, yod are used as self-referential and red, vdug are used as other-referential (as mentioned above, these auxiliary verbs and the endings they construct can also express other various grammatical meanings such as mood, tense and volition, etc.). Example (9) is a general statement using yovo red as the auxiliary verb.

In addition, the auxiliary verbs of TAM ending also have the function of indicating the source of information. For example, yin and yod specify that the event is egophoric, red and yovo red imply that the information may be a general truth or a specific fact, and $v d u g$ signifies that the speaker personally observed the fact or event. (Tournadre \& Lapolla, 2014)

There is a special case of ending. The auxiliary yod, vdug (or bzhag) and yovo red can be directly connected to the past tense form of the main verb to signify that the effects of an action performed in the past are still relevant at the moment when the statement is made. These endings are made up of only one auxiliary verb without any other components. Formally, it is obviously different from the structure of END 2 and on the contrary, very similar to the END 1. But Denwood (1999:116) made the point that this is the omission of the additional component $p a,{ }^{\mathbb{1}}$ which may be regarded as the zero form or a variant of $p a$. By this interpretation, he unified this form with END 2, and expediently made the usage of the past marker $p a$ keep consistent (that is, in this way, the markup of the past form can be

\footnotetext{
(1) In the original text, Denwood used the term "connecting partical".
} 


\section{The Structure and Hierarchy of Sentence-endings in Tibetan Language}

uniformly summed up as $p a$ ). We generalize it to a special form of END 2:
(10) zla nyin da tshod nga lha sar bsdad-yod
last year this time I Lhasa-LOC stayed-AUX
'I have already stayed in Lhasa at this time last year.'
(11) chos vphel-gyis nang-la yige gcig btang-yovo red
Chos vphel-GEN home-LOC letter one sent-AUX
'Chos vphel has sent a letter to home.'
(12) rgan lags-kyis ngatso bsgugs bzhugs -shag
teacher HON-AGE us waited stayed.HON -AUX
'The teacher has been waiting for us.'

\subsubsection{Moral ending, additional component is rgyu}

The second one (marked as END 3) consists of the additional component rgyu and the auxiliary verb yin, red, dug, yovo red. The verb that precedes the ending should be in the present-future. All these endings have a moral sense. Among them, rgyu yin and rgyu red indicate the future. They are practically the same as gi yin, gi red, but with more of a sense of obligation; in certain contexts, they may also imply that something that ought to have been done already has yet to be accomplished. Sometimes, rgyu red also means an action that is objectively required or prescribed (Hu Tan, 1999; Tournadre, 2003). rgyu yovo red and rgyu vdug mean "one should", but they appear more often in the negative form such as rgyu yovo ma red and rgyu mi vdug, and give the sentence some polite imperative tone to phrase a command or a request. For example:

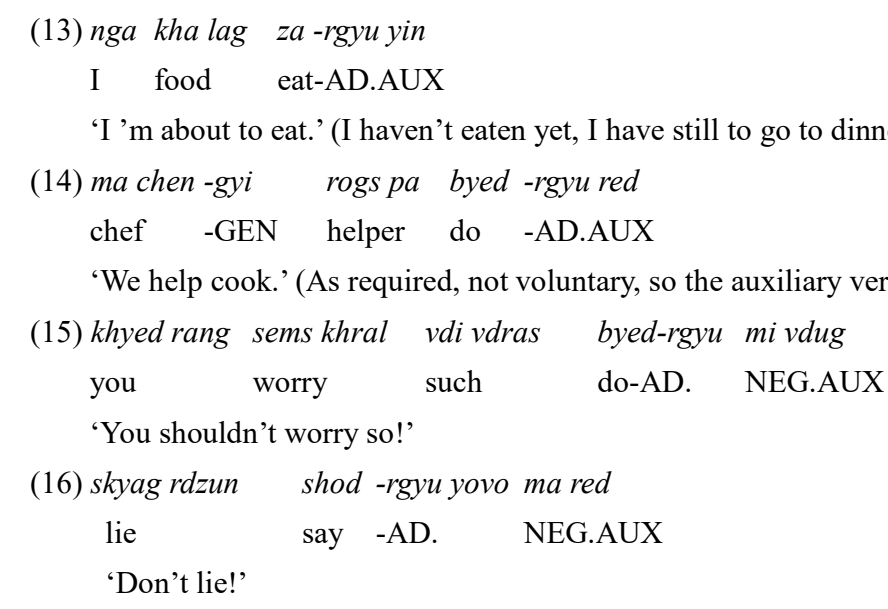

There is a similar ending tog med 'don't do something' whose structure and source are worthy of further study.

\subsection{Extended ending}

The third type of endings appears after the semantically self-sufficient sentence 
(including equational sentence and existential sentence, which are not constructed by the endings mentioned above). Formally, it is the ending occurring after the sentence ending rather than the verb phrase, or after the copula if the sentence is an equational sentence or existential sentence. For the convenience of expression, we call the ending which belongs to the self-sufficient sentence as the basic ending (as the forgoing two types), and this type which comes after the basic ending as the extended ending. Thus, the sentence structure pattern of this type of ending is:

lexical verb stem/predicate noun + basic ending (additional component/zero form + auxiliary) /copula + extended ending

In the light of its grammatical function and connection mode, this type can be divided into four sub-classes.

\subsubsection{Reported ending}

The first one (marked as END 4) is za (zag) or zer gyi za. It comes after the basic ending or copula, representing that the sentence information comes from someone's report or hearsay. When reporting the information, this structure is formulated directly without introducing a speaker or a source, which is different from the common quoted sentence. $z a$ is derived from the grammaticalization of speech verb zer 'to say', its meaning is equivalent to 'it is said that', and zer gyi za is equivalent to 'one is said to have said that'. Examples are as follows:

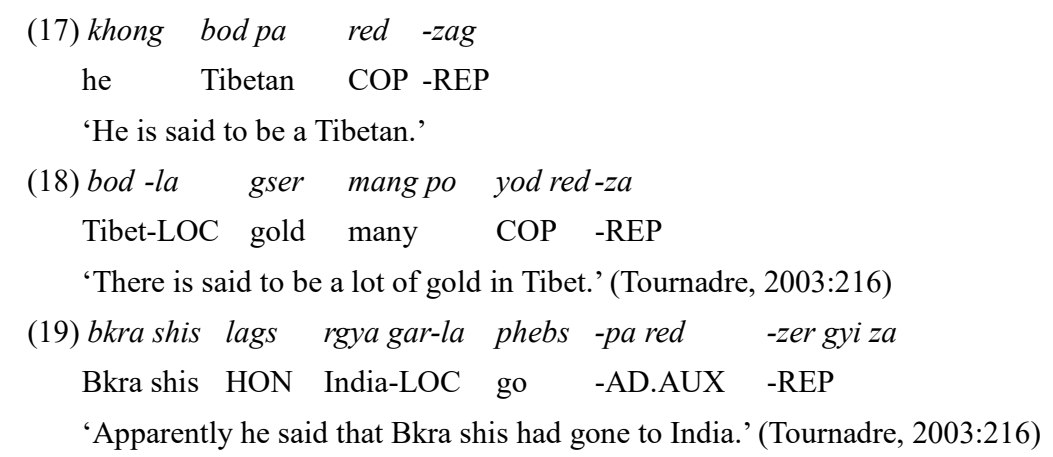

\subsubsection{Inferred ending}

The second one (marked as END 5) is pa yod, gyi red, sa red, pa vdra, mdog kha po red $^{\mathbb{1}}$. They are mainly connected behind END 2 or copula ${ }^{2}$, indicating that the information

\footnotetext{
(1) The difference in structure between mdog kha po red and other similar endings of this kind is that the additional component $m d o g$ kha po is a grammaticalized phrase rather than a suffix.

(2) Tournadre (2003:175) called these types of ending as "auxiliaries of probability", of which he referred to those ending structures connected with the copula as "epistemic copula", and those connecting with the auxiliary of basic ending as "epistemic auxiliary". In addition, some endings such as pa yod, pa vdug can also be used directly after the verb phrase. For example: (a) khong sang nyin tshogs vdur phebs-pa yod. (he tomorrow meeting-LOC come.HON-INF, 'I know he should come to the meeting tomorrow.'); (b)
} 


\section{The Structure and Hierarchy of Sentence-endings in Tibetan Language}

is indirectly inferred by the speaker. On the basis of varying contexts, these endings may be varied by the use of adverbs such as "surely", "probably" or the modal verb "must". Note that in this pattern, the auxiliary verbs of the basic ending or the copula are all in the self-referential form. Illustrations are as follows:

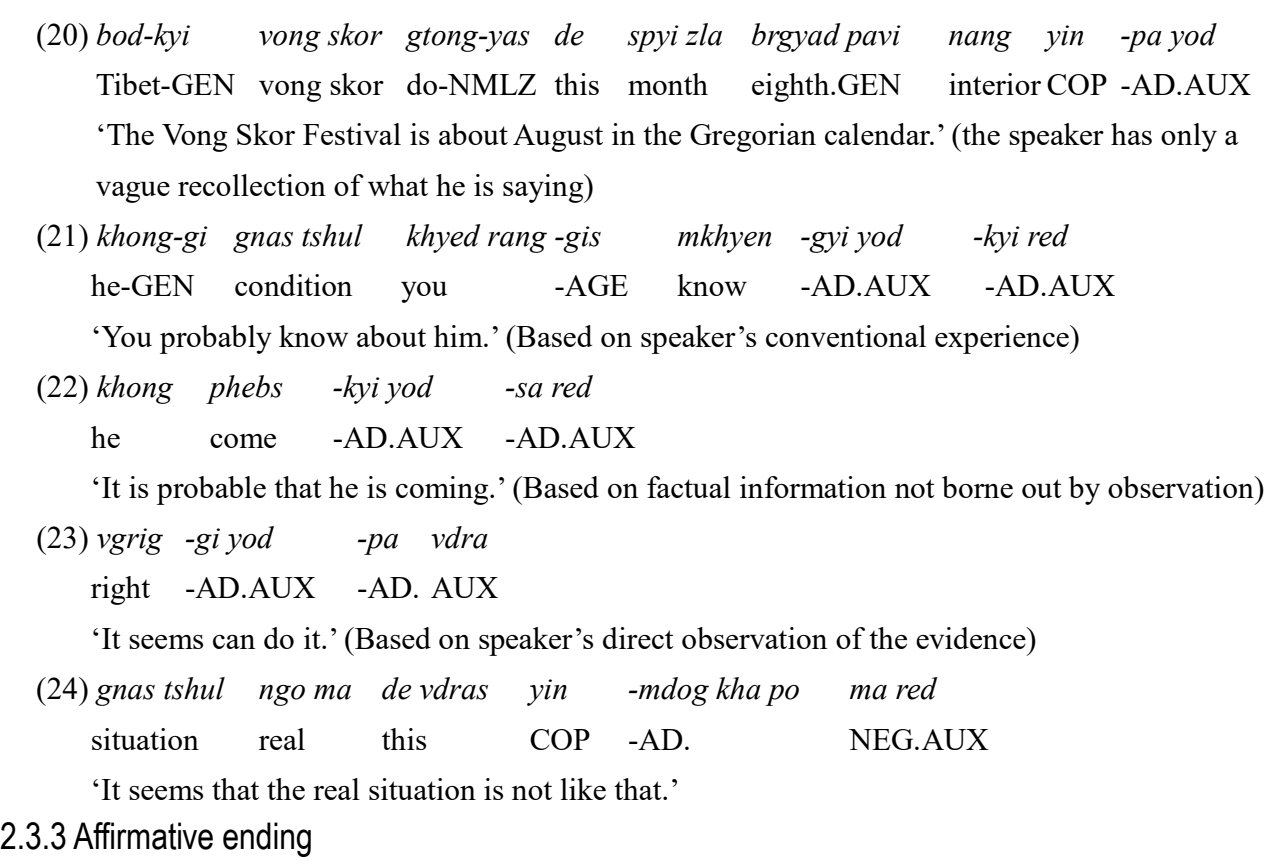

The third one (marked as END 6) is different in the way it is constructed. Or it is formed by the conjunction da/dang and the auxiliary verb yin/yod, such as (yin) da yin, (yod) dang yod. Together with the auxiliary verb of the basic ending (or the copula of equational sentence and existential sentence), it is also considered to be the overlapping form of the preceding auxiliary verb (or copula), representing the highest degree of confirmation. Or it comes from the grammaticalization of the quantizing structure located at the end of sentence, which forms a fixed ending structure with preceding auxiliary verb, such as (yin) shas che indicating the affirmation of a higher magnitude. Or it is an auxiliary verb such as vgro or vgrovo which is combined with the preceding auxiliary verbs to form yin vgro, yod vgrovo and similar ending structures. Their negative counterpart min vgro and med vgrovo occur more frequently than the positive auxiliaries. This kind of ending often constructs a fixed structure with the basic ending or copula, and like END 5, whose auxiliary verbs of basic ending are all in the self-referential form. For instance:

khos gnam gru rjes zin pa vdug. (he-AGE airplane catch-INF, 'He will certainly arrive in time for his flight.'); (c) rang cham pa brgyab pa vdra. (you cold had-INF, 'you seem to have a cold.'). 


\section{Li Huaping}

(25) ngavi phayul nyingkhrir yovored yul ljongs mdzespo yod -dangyod

I.GEN hometown nyingkhri.LOC COP scenery beautiful COP -CON.AUX

'My hometown is in Nyingchi, the scenery is of course beautiful.'

\begin{tabular}{|c|c|c|c|c|}
\hline $\begin{array}{l}\text { (26) slob sbyong } \\
\text { study }\end{array}$ & $\begin{array}{l}\text { yag-pa } \\
\text { good-NMLZ }\end{array}$ & $\begin{array}{ll} & \text { tshos } \\
\mathrm{Z} & \text { PLU.AGE }\end{array}$ & $\begin{array}{l}\text { slob sbyong-la } \\
\text { study-LOC }\end{array}$ & $\begin{array}{l}\text { dkav ngal } \\
\text { difficult }\end{array}$ \\
\hline yod-mkhan & tshor & rogs ram byed & $-r g y u$ & yin $\quad$-da yin \\
\hline have-NMLZ & PLU.DAT & help & -NMLZ this & COP -CON.AUX \\
\hline
\end{tabular}

'It is of course that good students help those who have difficulty in studying.'

(27) khong tshong pa yin -shas che

he businessman COP -QUA

'He is mostly a businessman.'

\subsubsection{Responsive ending}

The fourth one (marked as END 7) embodies two members. One is pa red, which is composed of the additional component $p a$ and the auxiliary verb red. This so-called "self-corrective" ending implies that the speaker has just realized that he was mistaken or that he was hitherto unaware of what he was asserting (Tournadre, 2003; Oisel, 2017). In this phenomenon, the auxiliary of the basic ending or copula is self-referential. The other one is red shag, factually, which consists of the auxiliary verb shag and a preceding copula or auxiliary verb which should be red rather than yin. Here red shag indicates that the consequence of an event is suddenly discovered by the speaker and contains some emotion of the speaker (shag can also directly follow the past tense of the verb, implying that the speaker is making an inference in discovering the result or the enduring traces of a past action). In most of the time, these two structures may be translated into English by adding "Oh!" "Why!" or "Look!" to the beginning of a statement in the past tense. Examples are as follows:

(28) khong las byas pa yin -pared

he cadre COP -AD.AUX

'Oh! He turned out to be a cadre!' (Now I see)

(29) nga gnyis-kyi nyal khang skya vbrel red-shag

I two-GEN dormitory neighbor COP-AUX

'Why! Our dormitory is actually next door!' (Unexpectedly)

Having classified the types of endings above, we will discuss the expressive functions of these endings and the syntactic levels to which they are related. Our discussion will be established on the perspective that, the semantic functions of ending are different, and correspondingly, the syntactic levels which the endings are associated with are also diverse. 


\section{The Structure and Hierarchy of Sentence-endings in Tibetan Language}

\section{The hierarchical study of sentence-endings' functions}

Language is a hierarchical structure. Van Valin \& Lapolla $(2002: 31,49)$ held the view that, the hierarchical structure of a sentence consists of three levels, nucleus, core and sentence ${ }^{\mathbb{1}} \ldots$ Of various grammatical categories, aspect, negation and direction act on the nucleus level; direction, modality and negation act on the core level; status ${ }^{2}$, tense, evidentials and illocutionary force ${ }^{(3)}$ act on the sentence level. Succinctly stated, from the point of view of grammatical units, these categories mainly act on three syntactic units respectively: predicate verb, verb phrase and sentence. The endings of the Tibetan language reflect different grammatical categories and function at different levels, and each is described below.

\subsection{END 1 acts on the predicate verb}

The END 1 primarily represents the category of aspect ${ }^{\oplus}$, which mainly acts on the predicate verb. From the semantic evolution of the endings, they were originally used as the second verb to supplement the meaning of the main verbs ${ }^{(5)}$. Even after grammaticalization, they still retain the semantic supplement of the predicate verbs to varying degrees. For instance, byung and song refer to the direction of action, shag implies a decisive act leading to a lasting state of affairs, sometimes with a pejorative overtone, and myong means that the action has been experienced, etc. Therefore, they principally act on the predicate verb. What is more, as far as its primary meaning is concerned, the END 1 cannot be followed by a modal verb. ${ }^{\odot}$ As for modal verbs, Lü Shuxiang (1979:110) once pointed out that modal verbs can be analyzed as "the higher-level predicate". Afterwards,

\footnotetext{
(1) The nucleus level mainly refers to the predicate part; the core level refers to the predicate structure and the core argument (agent, patient, etc.), namely the phrase part; the sentence level consists of the core and the modifiers outside the core (tense, aspect, voice, etc.). In other words, these three levels refer to predicate verbs, predicate verb phrases and sentences, respectively.

(2) State refers to two forms of representation of proposition: realistic and unrealistic.

(3) Illocutionary force mentioned here refers to the mood of a sentence, including declarative, interrogative and imperative, etc., which fulfills different communicative tasks with varying levels of performative force: declarative is used for providing information, interrogative for asking for information, and imperative for offering or requesting services.

(4) END 1 and END 2 also encode different types of evidentials, person, cognitive modality and other information. These semantic categories act on the phrase and sentence level. Here we only focus on their basic functions.

(5) Dalency (1991:13) stated that "serialization construction is a device which permits the use of two verbs to describe different aspects of a single event." When the second verb in the serialization structure is vacated, it still plays a supplementary role to the main verb, and its meaning includes the tendency, progress and state of the action. Denwood (1997:172) also noted, "as serial particles byas and nas indicate simultaneity of the actions of the two verbs, the first verb qualifying the second as a sort of 'adverb of manner"'.

${ }^{6}$ For example, when song is used in the extended meaning, it can occur after the voluntary verb dgos or even dgos byung. This is beyond the scope of this paper.
} 
Li Linding (1990:251) elaborated on this viewpoint emphasizing that "the modal verb is related to the whole sentence except for itself." From this point of view, we can say that the syntactic level of END 1 is below that of the modal verb, and its basic function does not apply to the whole sentence. In addition, it can be followed by an interrogative modal particle to convey the interrogative mood, but cannot be made into an imperative sentence by adding imperative particles, indicating that the END 1 is indicative.

\subsection{END 2 and END 3 act on the verb phrase}

END 2 and END 3 have the uniform structure type and both possess the function of sentence-completing. In END 2, the nominalized suffix (what we call "additional component" in this paper) " $g i$ can be regarded as 'imperfective', roughly equivalent to the English present participial '-ing', and $p a$ or its zero form can be regarded as 'perfective', roughly equivalent to English past participial "-ed" (Denwood, 1999:116). As for the copula (which is what we call "auxiliary verb" because of their grammaticalization. As mentioned earlier, there are two sets of auxiliary verbs: equational and existential), "the meaning of the first set (yin, red) is negative temporal accessibility; and the second set (yod, yod red, vdug) is positive temporal accessibility" (Delancey, 1990:289). When the nominalized suffix and copula are combined together, they basically express TAM meaning (as well as the meaning of categories such as person, evidentials and mood), but separating their contribution to reflect meaning might be intractable. Let's look at their function from the perspective of sentence formation. Much of the research in cognitive psychology suggests that in the experience of the real world, people obtain a series of event schemas through cognitive processing such as abstraction and categorization. An event schema is the abstraction of a concrete event and the description of the essential attribute of the event, but it ignores many details. Thus, if we want to talk about a specific event in actual language communication, we need to position it in the real world from three aspects of spatial, temporal (or TAM) and cognition (Zhang Lifei, 2012:68). TAM category is the principal component to locate the event schema of an abstract phrase, which makes a verb phrase turn into a communicative sentence and acts on the predicate phrase level. What is more, in the sentence of habitual aspect (gi yod, gi vdug, gi yovo red), it can also occur after a modal verb. According to the aforementioned standpoint that "auxiliary verbs are higher-level predicates", and the difference in the possibility of combining END 1 and END 2 with modal verbs, we can see that END 2 has a higher level than END 1. As for the communicative tone, END 2 conveys declarative mood, followed by the interrogative modal particles to express interrogative mood, but not the imperative particles, indicating that END 2 can be described as indicative as END1.

In END 3, the additional components rgyu are the nominalizer of verb phrase, representing the behavioral event itself; the auxiliary verbs red, med, and yovo ma red are 


\section{The Structure and Hierarchy of Sentence-endings in Tibetan Language}

used to make moral and rule judgments about an action or event formed by nominalizing structure $^{\mathbb{1}}$, and construct a sentence at the same time. That is to say, END 3 is the positioning component of event schema in deontic modality, which is the same as END 2, acting on the verb phrase.

The above three types of endings form a syntactic ladder from nucleus to verb phrase (END $1<$ END 2/END 3). What they have in common lies in that one of them must appear in order to turn a verb phrase into a sentence, and they all belong to the category of sentence-constructing and are therefore mandatory sentence-endings. END 1 mainly acts on the predicate verb's part, while END 2 and 3 mainly act on the verb phrase. The difference between END 2 and END 3 lies in that the former locates the event schema from the perspective of spatiotemporal, while the latter from the deontic modality. The former constitutes a realistic sentence, while the latter constitutes a non-realistic sentence. As far as communicative tasks are concerned, the sentence formed by END 3 is not to provide information, but to impose a certain behavior on the listener. Consequently, its illocutionary force is stronger than that of the END 1 and END 2.

\subsection{END 4 - END 7 act on the sentence}

END 4 - END 7 are the endings that follow the self-sufficient sentence and are the extended endings that follow the sentence. Generally speaking, components that are semantically grouped together are also syntactically placed together. As we will see below, such endings can form a semantic aggregation as they are all related to the self-sufficient sentence information, but with varying degrees of relevance.

END 4 belongs to the category of the evidentials, which is the speaker's hint to the source of the sentence information. The evidentials are characteristic of higher-level predicates, and the whole sentence has a hidden level of expression above the main stem: "I heard (someone or on the radio ...) that ..." The information source is not one of the contents of the information itself, but only a relevant element, for the ending is just an additional explanation.

END 5 belongs to the category of measure, which indicates that the information is inferred by the speaker based on the evidence implied by the ending; some scholars (Oisel, 2017) regarded it as a sub-category of the evidentials. Compared with the typical evidentials, this type of ending connotes the evidence indirectly or implicitly, such as the trace of events ( $p a v d r a$ ), conventional experiences ( $g y i$ red), faint memories ( $p a y o d$ ) and so on. The logical correlation between indirect evidence and information is relatively loose. Consequently, they are not adequate to confirm the authenticity of information. Therefore,

\footnotetext{
(1) Denwood (1999:233) pointed out that "a verb nominalised by rgyu means 'one should"'. Tounadre (2003:178) also pointed out that "verbs followed by the nominalizer rgyu correspond to the future or the imperfective, often with a sense of obligation".
} 
the information content of a sentence can only be measured by the speaker, and the meaning of the whole sentence is roughly equivalent to "(from the perspective of ...), it is probably ...". At this time, the whole sentence carries subjectivity.

END 6 belongs to the category of cognitive modality, indicating the speaker's assessment of whether the information is true or not. It is well-known that any information is either true or false objectively, and there is no intermediate state. But sometimes in the conversation, when the information of a sentence is not confirmed or needs to be emphasized, the speaker can estimate the polarity (positive or negative) of the sentence with different magnitude. Let's take the polarity of positive for example. In Tibetan, there are some diverse magnitudes of values such as dang yin 'of course is', thag chod yin 'absolutely is', vgro 'probably is', yin shas che 'mostly is' and so on. All of these are different values at the positive pole. The polarity is the internal factor of the information itself, but its estimate comes from the speaker, so END 6 is obviously subjective as well.

END 7 indicates that the information of the sentence is new. Unlike the evidentials, it carries the cognitive response of the speaker; some scholars (Delancey, 1997; Aikhenvald, 2004, 2012) generalized it to a category of mirativity. In the field of psychology, the four processes of cognition, emotion, volition and behavior are tightly correlated. In conversation, when the speaker finds his or her lag about the information, or when the new information exceeds the speaker's expectation, or when the speaker realizes his or her misunderstanding about the information, some cognitive emotions such as surprise, excitement, regret, doubt and even embarrassment arise spontaneously, and are reflected through the ending of the sentence. Such sentences with this kind of extended ending are also frequently used alongside cognitive interjections. The emotional reaction to information generally varies from person to person. People with different personalities and different information reserves often react variously to the same information, so the meaning of these endings is of personal subjectivity and not inevitably correlated to the information itself.

In a word, the semantics of the END 4-7 act on the whole self-sufficient sentence, which are the speaker's personal expressions. It is undeniable that on some occasions, when the speaker says a sentence, his personal expressions such as hint, inference, evaluation and reaction to the sentence information might be attached to the sentence. However, as analyzed above, different personal expressions have different correlations with sentence information. The source of the information hinted by the END 4 is the external element of the information rather than the information itself; the indirect and fuzzy evidence implied by the END 5 is not necessarily related to the content of the information, but only related to each other through the speaker's experience; the magnitude of polarity indicated by END 6 is the speaker's subjective cognition in that there is no magnitude about the polarity of 


\section{The Structure and Hierarchy of Sentence-endings in Tibetan Language}

sentence but only two poles objectively, i.e. positive and negative, so it doesn't belong to the sentence information itself; END 7 is a reflection of the speaker's subjective emotion, and has nothing to do with the information itself. The lower the degree of correlation between endings and clausal information is, the higher the corresponding semantic category and the speaker's subjective participation are, and the gradient of hierarchy from low to high is:

END $4<$ END $5<$ END $6<$ END 7

Additionally, unlike END 1-3, END 4-7 are neither necessary for the sentence nor the category of sentence-completion, so they are selective.

\subsection{Tibetan sentence-endings is a hierarchical system}

Tournadre (2003:175) once talked specifically about the copulas and auxiliaries of probability of the Tibetan sentence-endings. He pointed out that:

"In addition to final auxiliary verbs that convey various tenses and evidential moods, there is also a set of 'epistemic' final copulas and auxiliaries that expresses different degrees of certainty or probability .... These auxiliaries are also associated with different tense-aspects, and for the most part involve the same verbs as the evidential auxiliaries, but combined in a different way."

Here, the "epistemic final copulas and auxiliaries" mentioned by Tournadre mainly refer to END 5 in the third type of this paper, but the other endings such as END 4 and END 6-7 in this category are also very different from the first two categories in terms of syntactic distribution and function. Based on the study of Tournadre, Lim divided the endings of sentence into two parts: $\mathrm{TAM}_{\mathrm{A}}, \mathrm{TAM}_{\mathrm{B}}$, and the proposed morphological template for the TAM suffixes are as below:

Table 1. Morphological template for the TAM suffixes

\begin{tabular}{c|c|c|c|c}
\hline & \multicolumn{2}{|c|}{$\mathrm{TAM}_{\mathrm{A}}$} & \multicolumn{2}{c}{$\mathrm{TAM}_{\mathrm{B}}$} \\
\hline $\mathrm{V}_{\text {stem }}$ & $\left(\mathrm{CP}_{1}\right)$ & $\mathrm{TAM}_{1}$ & $\left(\mathrm{CP}_{2}\right)$ & $\mathrm{TAM}_{2}$ \\
\hline
\end{tabular}

(Adapted from Lim's work (2012))

He (2012:11) delivered this template a further explanation. He noted that:

"The first portion or the inner TAM suffixes, TAMA, is the same as the regular TAM suffixes, but only indicate tense in the probability construction ......The secondary connecting particles -ki or -pa are required when there are two suffixes following a verb stem. The second portion or the outer probability TAM suffixes, TAM $M_{B}$, indicate both the evidential and the confidence level or epistemic modality of a proposition."

The endings belonging to TAM which Lim refers to are roughly the same as those of Tournadre's, but he separated the epistemic endings from the basic ending on the syntactic and sematic levels, and pointed out the pragmatic meaning of the evidentials and confidence in the epistemic endings, which expanded the scope of the epistemic endings to 
a certain extent.

According to our previous analysis, from the perspective of syntactic distribution and semantic function, the other endings (END 4 and 6-7) of the third type also greatly differs from the first two types, and belong to the outer part of the endings just like END 5. From this perspective, we can divide the sentence-endings of the Tibetan language into two parts: the first part mainly expressing TAM meaning belongs to the sentence-completion category; the second part mainly expressing evidential and cognitive modality belongs to the pragmatic category (see Figure 1). And then, according to the corresponding syntactic level and the degree of speaker's subjective participation, the hierarchy gradient of ending is ranked from low to high in the following order:

Sentence-completion $($ END1 $<$ END2/END3) $<$ Pragmatic $($ END4 $<$ END5 $<$ END6 $<$ END7)

We denominate self-sufficient sentences constructed with END 1-3 as syntactic sentence and sentences which also constructed with END 4-7 simultaneously as pragmatic one.

Finally, it should be added that all the endings' functions mentioned above are typical functions of the Tibetan endings. In fact, in a certain communicative environment, these basic functions of low-level endings will be expanded and become a form of discourse with the function of subjective expression, which deserves further study.

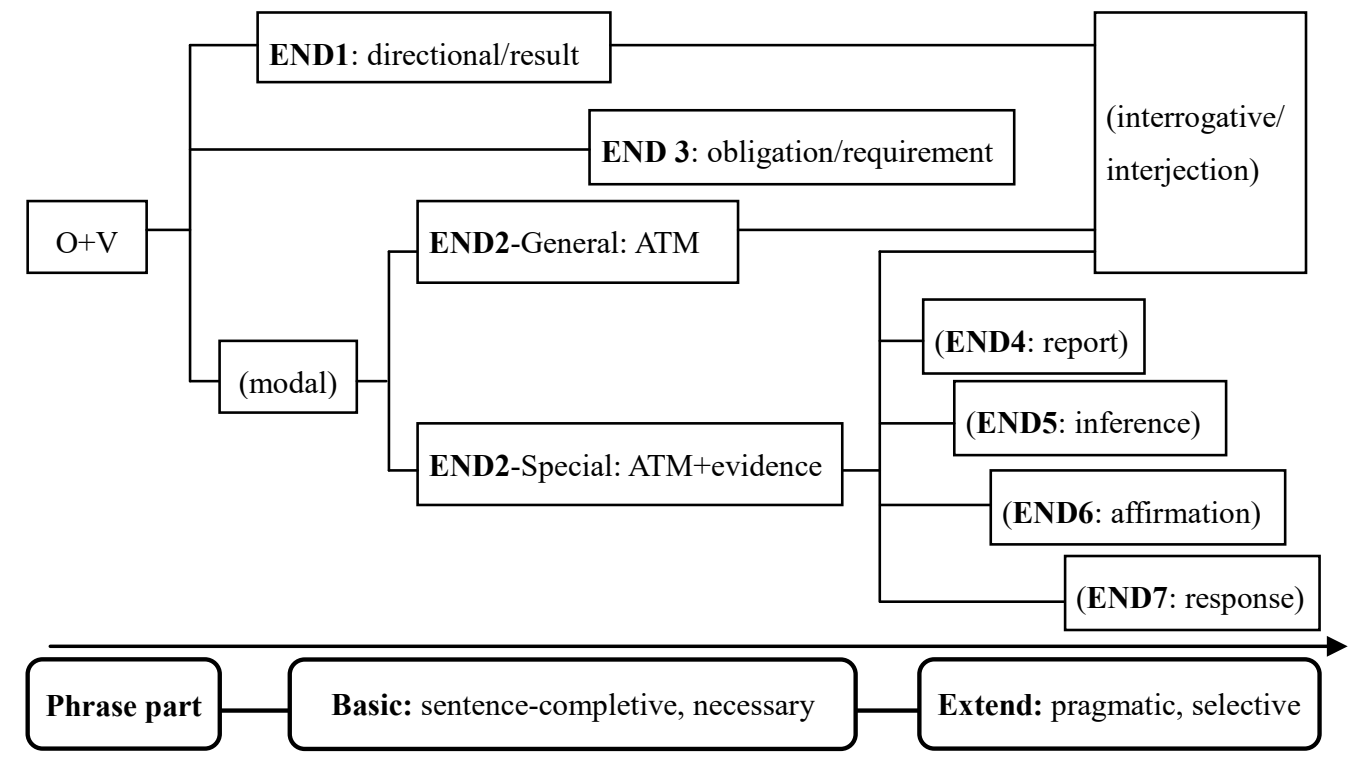

Figure 1 . The hierarchy of Tibetan sentence-endings

\section{Abbreviations}

$\mathrm{ABL}$

$\mathrm{AD}$

AGE

Ablative

Additional Component

Agentive

$\begin{array}{ll}\text { AUX } & \text { Auxiliary } \\ \text { CON } & \text { Connective } \\ \text { COP } & \text { Copula }\end{array}$

Copula 


\section{The Structure and Hierarchy of Sentence-endings in Tibetan Language}

$\begin{array}{llll}\text { DAT } & \text { Dative } & \text { OTH } & \text { Other-Referential } \\ \text { END } & \text { Ending } & \text { PER } & \text { Perfect } \\ \text { GEN } & \text { Genitive } & \text { QUA } & \text { Quantizing Structure } \\ \text { GST } & \text { General Statement } & \text { REP } & \text { Report } \\ \text { HON } & \text { Honorific } & \text { SEL } & \text { Self-Referential } \\ \text { LOC } & \text { Locative } & \text { SM } & \text { Subordinate Marker } \\ \text { NEG } & \text { Negation } & \text { SPFV } & \text { Simple Perfective } \\ \text { NMLZ } & \text { Nominalizer } & \text { SST } & \text { Special Statement }\end{array}$

\section{References}

Aikhenvald, A. 2004. Evidentiality [M]. Oxford: Oxford University Press.

Aikhenvald, A. 2012. The Essence of Mirativity [J]. Linguistic Typology, 16(3):435-485.

Delancey, S. 1990. Ergativity and the Cognitive Model of Event Structure in Lhasa Tibetan [J]. Cognitive Linguistics, 1(3):289-321.

Delancey, S. 1991. The Origins of Verb Serialization in Modern Tibetan [J]. Studies in Language, 15(1):1-23.

Delancey, S. 1997. Mirativity: The grammatical marking of unexpected information [J]. Linguistic Typology, 1(1):33-52.

Delancey, S. 2011. Finite Structures from Clausal Nominalization in Tibeto-Burman [J]. Nominalization in Asian languages: Diachronic and typological perspectives, 1:343-360.

Delancey, S. 2018. Evidentiality in Tibetic [A]. In A. Aikhenvald (ed.). The Oxford Handbook of Evidentiality [C]. New York: Oxford University Press, 580-594.

Denwood, P. 1999. Tibetan [M]. Amsterdam; Philadelphia: John Benjamin's Publishing Company.

$\mathrm{Hu}$ Tan, et al. 1999. Primer of Lhasa Tibetan [M]. Beijing: Nationalities Press.

Hu Tan. 2002. Studies on Tibetan Language [M]. Beijing: China Tibetology Press.

Jiang Di. 2005. Aspect, Evidentiality and Egocentricity in Lhasa Tibetan [J]. Linguistic Science, 4(1):70-88.

Jin Peng. 1979. On the Relationship Between the Grammatical Structure and the Characteristics of Tibetan Spoken Verbs in Lhasa [J]. Minority Languages of China, 3:173-181.

Jin Peng. 2001. Tenses and Expressions of Modern Colloquial Verbs in Tibetan [A]. In Jin Peng. Collection of Ethnic [C]. Beijing: National Press, 253-300.

Li Linding. 1990. Overview of Verb Classification Research [J]. Chinese Language, 4:248-257.

Lim, J. 2012. The Verb System of Standard Tibetan [OL]. Download website: https://www.academia.edu/ 4661633/2012_COMPII_Verb_of_Lhasa_Tibetan.

Lü Shuxiang. 1979. Analysis of Chinese Grammar [M]. Beijing: Commercial Press.

Oisel, G. 2017. Re-evaluation of the Evidential System of Lhasa Tibetan and its Atypical Functions [J]. Himalayan Linguistics, 16(2):90-128.

Skalbzang vgyurmed. 2004. A Course in Practical Tibetan Grammar [M]. Chengdu: Sichuan Publishing Group, Sichuan Nationalities Publishing House.

Tournadre, N. \& R. J. Lapolla. 2014. Towards a new approach to evidentiality: Issues and directions for research [J]. Linguistics of the Tibeto-Burman Area, 37(2):240-262.

Tournadre, N. \& Sangda Dorje. 2003. Manual of Standard Tibetan: Language and civilization [M]. New York: Snow Lion Publications, Ithaca.

Van Valin, R. \& R. J. Lapolla. 2002. Syntax: structure, meaning, and function [M]. Beijing: Peking University Press.

Zhang Lifei. 2012. The Cognitive Interpretation of Sentence Hierarchy [J]. Foreign Languages Research, 1: 64-68.

Zhou Jiwen \& Xie Houfang. 2003. Grammar of Tibetan Lhasa Dialect [M]. Beijing: Nationalities Press. 